\title{
Process Condition Monitoring - a Novel Concept for Manufacturing Management Tool Integration
}

\author{
J.M. Mendonça(1), B. Ribeiro(2), P. Silva(2) \\ (1) INESC-Porto/ DEEC-FEUP (2) INESC-Porto \\ Rua José Falcão 110, 4000 Porto, Portugal \\ Phone: +35122094300,Fax:+35122008487 \\ email:jmm@tecno.inescn.pt
}

\begin{abstract}
Manufacturing management requires increasing software and systems support for reactive decision making and optimised control of relevant performance parameters for improved competitiveness. This paper introduces and develops a novel concept, which is based on the use of a toolbox-based approach to the construction of manufacturing management solutions. Although this concept can be generally applied when implementing planning, scheduling and manufacturing control systems, the emphasis here is being put in the so-called shop-floor area. The first pilot implementation at a shoe manufacturing company is able to demonstration both the effectiveness and the still unexploited potential of the Process Condition Monitoring (PCM) concepts and tools.
\end{abstract}

Keywords

Condition Monitoring; Manufacturing Management; Manufacturing Execution Systems (MES); Shop-Floor Control Systems (SFCS).

\section{EMERGING REQUIREMENTS AND DEFICIENCIES OF EXISTING TOOLS}

Manufacturing management, particularly at shop floor level, requires software support for automatic factory data collection and processing, recording of historical information for further analysis, reactive decision making and optimised control of relevant performance parameters for improved competitiveness. These parameters relate to more traditional cost, quality and time issues, as well as to new aspects such as precision of delivery and enterprise reactivity or 
flexibility [Richards, 95]. This has been supported so far in a number of different ways, but mainly:

- through specific in-house or subcontracted developments leading to tailor-made solutions. The main advantage here is the adequacy to the specific company problems and manufacturing environment, and the associated drawbacks are the long development time and the large costs involved, these encompassing both the development cost and the cost of ownership;

- through standard packages configured to the specific company site. These are certainly cheaper and provide faster solutions, but they offer facilities often not adequately mapping the end-user requirements (offering and charging unnecessary functions while not meeting exactly the user requirements).

The inadequacies of both the development-based and the packaged-based approaches referred above, together with the emerging need for Information Technology (IT) systems support to new and more flexible organisational paradigms (cells, cooperative work, fractal organisations, etc.) and to novel production strategies (JIT, Kaizen, TQM, TPM, etc.), led to increasing requirements being imposed on software and systems.

In fact, heavy, monolithic, closed, vendor-dependent and department-oriented packages no longer satisfy most of the users, for their cost, burden on the organisation, rigidity and lack of adequate user interfacing (visualisation, information access, etc.). Furthermore, they are incapable of coping with fast changing organisation needs, multi-skilled users, requirements for information dissemination both across departments (horizontally) and at different hierarchical levels (vertically), and needs for decision synchronisation and control throughout the company planning, execution and control chains (particularly when decision power needs to be returned to the shop floor).

The answers to these problems are up to now only partial, with both the MRP-II and the SCADA (Supervisory Control and Data Acquisition) systems vendors starting to offer, respectively from the planning and from the process control level, solutions at the manufacturing execution or shop-floor control level. While the first ones seem to remain heavy and vendor-department packages, the second ones are just appearing in the market and it is still not clear what they will provide. Furthermore, both have not yet demonstrated their ability to help solving the complex, dynamic and often less structured environment that characterises the manufacturing execution level in many companies irrespective of their size or business [Mendonça, 1994], [Richards, 1995].

This paper introduces and develops a novel concept, which is based on the use of a toolboxbased approach to the construction of manufacturing management solutions. Although this concept can be generally applied when implementing planning, scheduling and manufacturing control systems, the emphasis here is being put in the shop-floor area. Further than that, the integration of aspects and functions traditionally "owned" by separate, independent departments, such as quality, maintenance, or product flow control, is a major issue of the PCM concept.

In order to achieve this, besides taking full advantage of state-of-the-art IT support, in terms of heterogeneous platforms and the use of standards "de facto" for operating systems, networking, data base access, client-server organisation, etc., i.e. the technological aspects, a structuring concept is needed to help selecting, building and integrating the right set of tools. 


\section{THE ROOTS OF PROCESS CONDITION MONITORING}

The concept of Process Condition Monitoring (PCM), which was developed and put into practice in the course of EP-8865 Real-I-CIM [Mendonça, 1995]*, builds on three well proven concepts:

- that of Machine Condition Monitoring (MCM), used in advanced instrumentation systems for monitoring and protecting critical or expensive machinery (e.g. turbines, pumps, etc.);

- that of Process Monitoring through SCADA systems used in continuous/batch process control (e.g. chemical, cement, pharmaceutical, food industries);

- that of Continuous Improvement (or Kaizen Engineering), particularly in what concerns shop floor performance monitoring for manufacturing process improvement through the socalled "seven tools" and analysis of historical data through statistical tools, data mining, etc.

\section{CONTROL LOOPS FOR LOCAL PERFORMANCE IMPROVEMENT}

The Real-I-CIM (RIC) PCM concept integrates real-time monitoring with advanced control at a lower (on-line/shop floor) level, and integrated performance monitoring and evaluation with intelligent data analysis and knowledge extraction at an higher (off-line/engineering) level.

Locally at the factory floor, PCM can be seen as undertaking watchdog/protective functions through real-time monitoring of relevant output/state variables and reactive management/control of relevant input/control variables of a manufacturing process resource or group of resources, e.g. a workstation, cell or line (Figure 1).

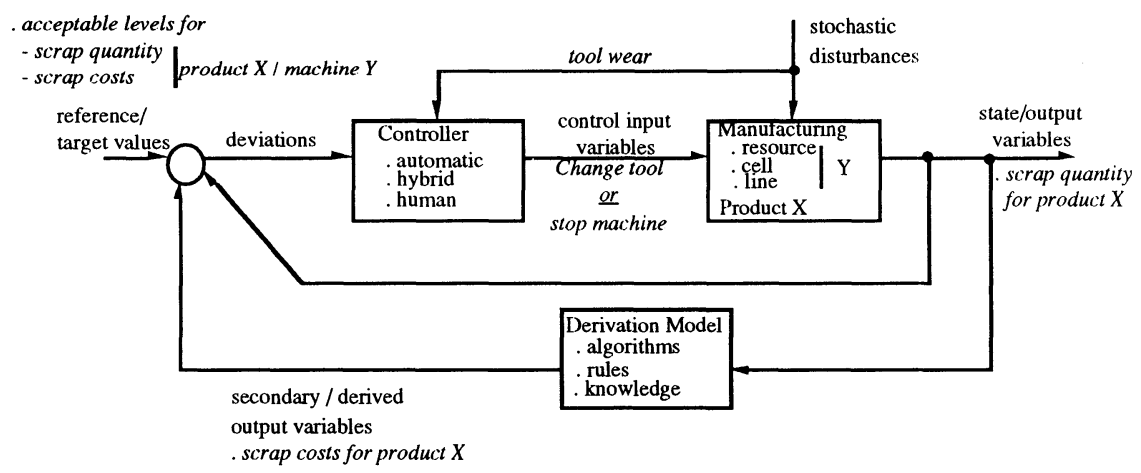

Figure 1 Local PCM Loops - real-time monitoring \& reactive management/control (example showing solely quality management functions).

* EP-8865 Real-I-CIM (Real-Interactive Shop-Floor CIM) is an european R\&D project co-funded by the Esprit programme which is targeted at the development and test of advanced tools supporting the implementation of low-cost solutions for short-term planning and control of discrete manufacturing processes. 
State/output variables characterising the process local performance are measured and compared with target or reference values, either established by the design or agreed through the analysis of historical records and/or heuristics. Other secondary, or derived, output variables may be then calculated by manipulating the first ones through so-called derivation models.

The lack of adjustment of both measured and/or derived variables to their reference values is then interpreted and possibly associated with synthoms and/or causes for the observed malfunctions or deviations to the desired operation target. These deviations are used by a controller to derive adequate corrective actions, these actions taking the form of changes to the control/input variables of the manufacturing process which aim to regain or re-establish control to the desired levels.

Figure 1 presents an example of a local real-time monitoring and reactive control loop within the scope of the quality function. These local PCM loops can be established for each individual manufacturing process unit (be it a workstation, a cell or a line), as to allow process control improvements supported or guided by relevant local performance indicators. Each of these units should be actually seen as an integrated whole comprising manufacturing resources (machines and operators) and material flow, together with information systems and information flow (e.g. manufacturing orders), and including the underlying organisational issues as well (Figure 2).

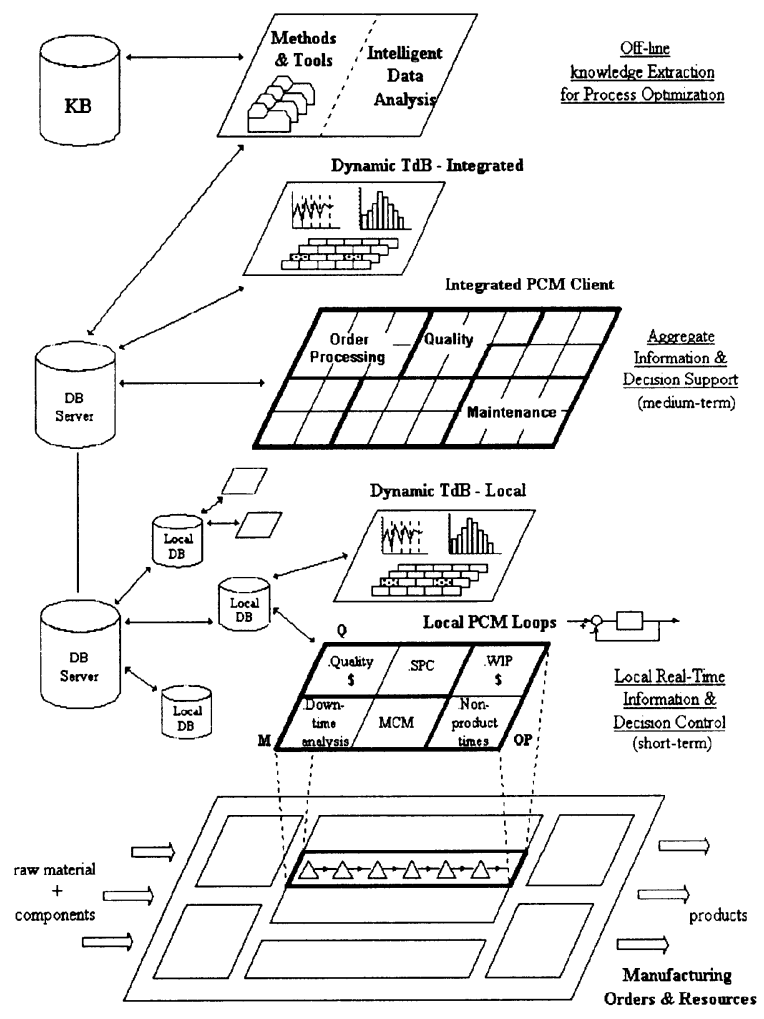

Figure 2 Interaction between different PCM components and tools. 


\section{KNOWLEDGE AS THE PRE-REQUISITE FOR CONTROL}

The controller at each loop can be classified as hybrid, manual or automatic, depending on wether its functions are undertaken by a human operator, assisted or not by adequate decision support software, or by a computer-based decision loop. Since usually a large number of different variables (causes) interact resulting in changes in a large number of other variables (synthoms) (Figure 3), the knowledge on the qualitative and/or quantitative relations between causes and effects has to support the evaluation of the observed deviations and the derivation of the corresponding corrective actions. The controller must therefore have some degree of embedded intelligence or knowledge, for example a set of rules in the simpler case.

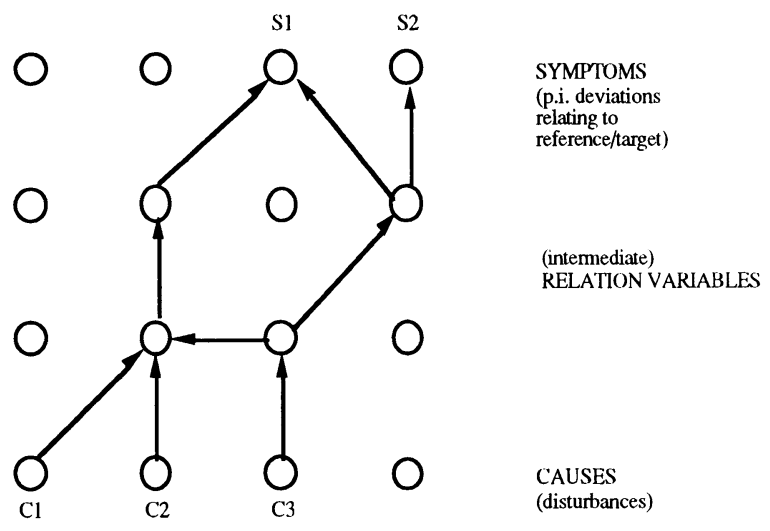

Figure 3 Interaction and interdependency between variables.

The above implies the need for information filtering and aggregation, as well as the need for knowledge extraction, at various levels (Figures 3 and 4). Logging all relevant events for further analysis, particularly each complete event chain formed by "change in output + deviation + evaluation + corrective action derivation + measured reactive change in process output", is of paramount importance to extract and structure crucial knowledge on the manufacturing process, that can be used to suggest corrective changes, e.g. to the machine set-up or operating procedures or even to the process itself.

\section{INTEGRATION OF PCM COMPONENTS AND TOOLS}

For monitoring and controlling purposes the PCM toolset includes modules performing shopfloor data collection, data logging and simple data analysis and interpretation, within the scope of the classical quality, maintenance and WIP/order tracking functionalities. The PROFIT**

** PROFIT (Production Optimization through Flexible Integrated Software Tools) is a toolset providing support to factory floor management in the scheduling, simulation, monitoring, quality and maintenance areas. It is being used in the RIC project as a prototyping tool. 
quality, maintenance and WIP tracking modules are being used to build, test and improve prototypes for the PCM toolset. The first real test beds for these prototypes are on-going within the scope of the so-called early RIC pilot site implementations (Growela, a portuguese shoe manufacturer, and one of BMW plants near Munich, Germany). They are being used to collect and process relevant information and to offer specific decision support and controlling functions within the scope of the specific users environment.

During the course of the RIC project these modules have also been improved with new functionalities, as to meet the industrial end-user requirements from the pilots and both the RIC Interest Group, formed by a number of manufacturing companies in Portugal and Germany, and the RIC marketing partners feedback. The facilities provided by modules traditionally belonging to department oriented packages, for example SPC being associated with the quality department and WIP tracking with production, will be here available in. a modular, readily configurable manner as to build PCM loops with integrated functionalities (Figure 2). These local real-time information and decision control loops will allow higher level integrated PCM clients to source information from the data base server providing aggregate information and decision support.

Information integration at an higher level will enable customised dynamic "tableau de bords" (DTBs) with business specific performance indicators to be configured from an existing library that can be continuously updated as needed. These DTBs will provide derived, more complex, semantically rich indicators both at local and more global level (Figure 2).

Finally, an ontological level will be superimposed through the use of knowledge-based (KB) tools, which will take advantage of the historical recordings with information from lower levels to extract, organise and improve the knowledge on the process, allowing further reference values, corrective actions and controlling strategies to be derived and deployed (Figure 4). This is in fact an area of growing interest in many industries, e.g. microelectronics or automotive, due to the ever increasing competitive pressures.

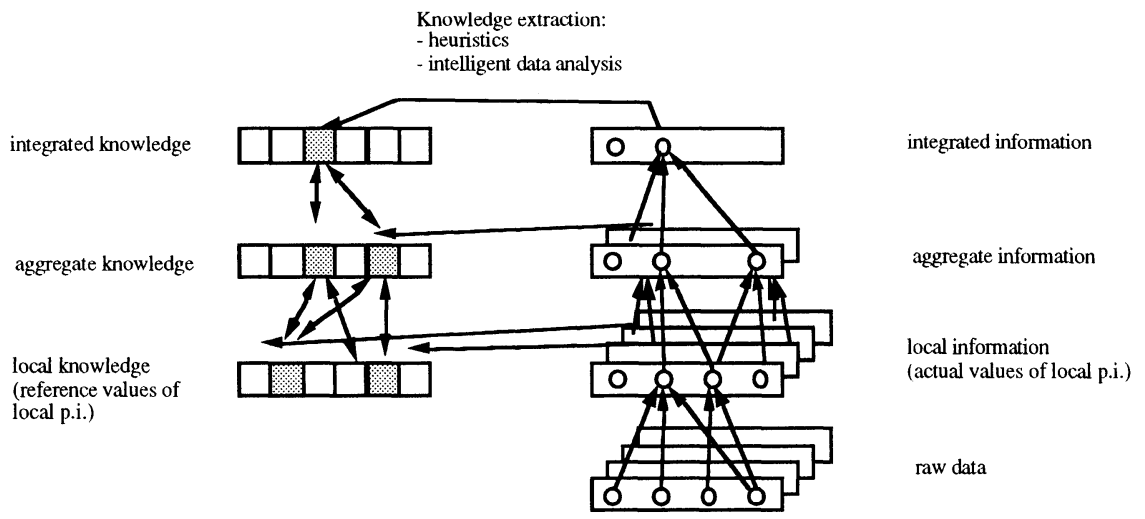

Figure 4 Hierarchized levels of information and knowledge extraction.

Within this context the use of $\mathrm{KB}$ should be looked at as a rather general concept, and the adequacy of different approaches and methods for "intelligent data analysis" is being investigated (classical statistical analysis, pattern recognition/feature extraction techniques, AI 
techniques, rule-based/neural fuzzy methods, constraint-based reasoning, genetic algorithms, data mining, etc.). Off-the-shelf tools running under Unix and MS-Windows are being used to build the underlying integration infrastructure, as it is discussed next.

\section{ARCHITECTURE AND INFRASTRUCTURE REQUIREMENTS}

Figure 2 presents an integrated view showing the interaction between the various PCM components and tools, from which it becomes evident the need for an advanced integrating infrastructure, the description and discussion of which falls out of the scope of this paper.

In fact, in order to support the PCM concept the RIC architecture and infrastructure had to meet a number of requirements which are briefly laid out and justified below.

- Modularity, as to allow low-cost entry points for an installation, the gradual growth of the installed system functionalities and an easier customisation of the available set of tools to the specific user needs.

- Flexibility, since the applications/tools built by the PCM toolset will have to interact with different external (legacy) company IT systems, like MRP-II, accounting or shop-floor data collection systems usually provided by different vendors. A highly flexible architecture is therefore necessary in order to make it possible to interact with such a wide variety of systems. Facilities to allow and ease the changing of the interfaces to these systems are also required.

- Performance - The PCM concept will naturally lead to a distributed set of applications running in several computers linked through communication networks and sharing a common logical data base. Fast communication and remote data access is thus a major requirement.

- Distribution - The PCM toolset includes a set of modules performing tasks relating to quality, maintenance, WIP tracking, etc., performing shop-floor data collection, as well as data logging and processing in different organizational units of the company. The system will therefore have to be distributed over various computers and terminals spread over the organisation.

- Openness - In order to achieve the above mentioned flexibility, i.e. an easily accessed and configurable system, an open infrastructure is necessary preferably supported by the use of "de facto" standards.

- Portability - The PCM toolset functionalities/tools should run in different vendor platforms, in at least Unix and MS-Windows environments. Therefore it is a major requirement that not only the tools and applications but also the infrastructure should be easily portable.

- Security and Reliability - The ability to provide continuously its services and to offer adequate recovery procedures is necessary. Simple security mechanisms should be provided as to ensure a levelled access to the system by the various user profiles, as well as to protect the system information and facilities from unauthorized users.

- Migration support - An important requirement of today's IT systems is that the process of evolving to new systems/platforms is easy and fast.

Details on the RIC architecture and infrastructure can be found in [Mendonça, 1995]. 


\section{IMPLEMENTATION METHODOLOGY AND EXPERIENCE}

The partial or complete implementation of the IT systems and tools discussed in the sections above can help companies in the development of their quality systems, in undertaking continuous improvement programmes or in re-organising manufacturing processes or process chains. However, the system potential benefits can only be obtained, and potential installation disasters avoided, if and adequate implementation methodology is used.

The toolbox approach to the development of integrated IT solutions and the distributed nature of these solutions, pervading different departments and hierarchical levels, absolutely require that the human and organisational aspects are taken into account. The factory implementation of the PCM tools should therefore start with requirements analysis work encompassing the so-called techno-organisational aspects [Soares, 1996].

Secondly, the system specification and development work should be supported by a prototyping strategy. This allows early feedback to the functionalities provided, high degree of customisation and easy user appropriation of the system. Since this should also be used to foster and support changes in the organisation and re-engineering of processes wherever appropriate, a stepwise approach with a progressively "wider and deeper" implementation of modules should be adopted, thus calling for a necessary prioritisation [Mendonça, 1995].

The experience gained so far with the pilot installation in Growela confirms and reinforces the need for such innovative implementation methodologies. Following to a detailed requirements analysis where techno-organisational aspects were covered, a basic set of core functions was first implemented: shop-floor interaction, production monitoring and selected quality and maintenance management functionalities. This set of applications share a common information model of the company manufacturing sections. This first prototype was then integrated with two major legacy systems: an existing shop-floor data collection system and the company planning system (MRP-II). The data which is collected at selected points along the production flow is further used for real time monitoring of resources and work-in-process (WIP) of manufacturing orders, quality tracking, breakdown management and for building performance indicators at various levels. This has enabled a very good insight into the details of manufacturing operations while providing line supervisors with basic decision support. The development of the second prototype, covering also short-term scheduling and dispatching as well as production prognosis facilities based on "what-if" simulations, is now being undertaken. Meanwhile, company specific performance indicators are been derived and organized under the form of DTB's that will be used at different levels in the hierarchy.

The first experiences of system operation was very positive, namely showing that:

- previous innacurate production monitoring, and consequent bad control of WIP, could be improved;

- faster reaction to quality problems and better supplier evaluation was possible.

The forthcoming steps will include the analysis and improvement of the order release system at the stitching lines (the so-called distribution problem), and to expand the scope of the pilot by increasing the number of data collection points and decision support terminals in the whole factory.

The Growela pilot implementation is therefore able to demonstrate both the effectiveness and the still unexploited potential of the PCM concept. 


\section{REFERENCES}

Richards, H.D., Besant, C., Mendonça, J., de Ridder, L. (1995) Planning, Scheduling and Manufacturing Control Systems - Final Report of the Esprit Project 9245 PASSE.

Mendonça, J.M., et al (1995) Yearly Technical Report of the Esprit Project 8865 Real-I-CIM.

Soares, A.L., Ferreira, J.J., Mendonça, J.M. (1996) A Tool for Techno-Organizational Design of Integrated Manufacturing Systems, Accepted for publication at the International Journal of Studies on Informatics and Control.

Mendonça, J.M., Schulte, J.W., and Távora, J. (1994) Production Planning and Control Systems and their Factory Implementation, 10th Esprit CIM - Europe Conference, Copenhagen, Denmark.

\section{BIOGRAPHY}

José Manuel Mendonça holds a degree in Electric Engineering by the University of Porto (1977) and received his $\mathrm{PhD}$. degree by Imperial College, London (1986).

His professional activities include Brown, Boveri \& C. ie, Switzerland (1977), Denmark's Technical Highschool (1978/79) and EDP - Portuguese Power Corporation(1979/80).

From 1980 he has a teaching position at the Faculty of Engineering University of Porto, being responsible for chairs in Quality Management, Computer Control and Manufacturing Systems Integration.

Since 1987 he is also a senior researcher at INESC (Instituto de Engenharia de Sistemas e Computadores) in Porto, where he is presently the head of the Manufacturing Systems Engineering Group. He has been responsible for numerous national and european R\&D projects, most notably Esprit projects 5478-Shop-Control and 8865-Real-I-CIM. 